(2) Open Access Full Text Article

\title{
Imaging choroidal neovascular membrane using en face swept-source optical coherence tomography angiography
}

This article was published in the following Dove Press journal:

Clinical Ophthalmology

13 October 2017

Number of times this article has been viewed

\author{
Magdy Moussa ${ }^{1,2}$ \\ Mahmoud Leila ${ }^{3}$ \\ Hagar Khalid ${ }^{1,2}$ \\ 'Ophthalmology Department, \\ Faculty of Medicine, Tanta University, \\ Tanta, Egypt; ${ }^{2}$ MEDIC Eye Center, \\ Tanta, Egypt; ${ }^{3}$ Retina Department, \\ Research Institute of Ophthalmology, \\ Giza, Egypt
}

Purpose: The aim of this study was to assess the efficacy of swept-source optical coherence tomography angiography (SS-OCTA) in delineating the morphology of choroidal neovascular membrane (CNV)

Patients and methods: This was a retrospective observational case series reviewing clinical data and fundus fluorescein angiography (FFA), swept-source optical coherence tomography (SS-OCT), and SS-OCTA images of patients with CNV and comparing the findings. The sweptsource technology enables deeper penetration and superior axial resolution. The incorporated blood flow detection algorithm, optical coherence tomography angiography ratio analysis (OCTARA), enables visualization of CNV in vivo without the need for dye injection.

Results: The study included 136 eyes of 105 patients. Active lesions on SS-OCTA images showed increased capillary density, extensive arborization, vascular anastomosis and looping, and peri-lesional hollow. Inactive lesions showed decreased capillary density, presence of large linear vessels, and presence of feeder vessels supplying the CNV. We detected positive correlation between SS-OCTA, FFA, and SS-OCT images in $97 \%$ of eyes. In the remaining 3\%, SS-OCTA confirmed the absence of CNV, whereas FFA and SS-OCT either were inconclusive in the diagnosis of $\mathrm{CNV}$ or yielded false-positive results.

Conclusion: SS-OCT and SS-OCTA represent a reproducible risk-free analog for FFA in imaging CNV. SS-OCTA is particularly versatile in cases where FFA and SS-OCT are inconclusive.

Keywords: swept-source OCT, OCT angiography, imaging of CNV, OCTARA algorithm

\section{Introduction}

To date, fundus fluorescein angiography (FFA), indocyanine green angiography (ICG), and spectral-domain optical coherence tomography (SD-OCT) remain the benchmark imaging modalities in the diagnosis of choroidal neovascular membrane (CNV) and the gold standard against which the efficacy of emerging technologies is checked. A major advantage of FFA and ICG in imaging CNV is their ability to capture real-time transit of injected contrast through retinal and choroidal vasculature and relay information on dye transit in the form of specific patterns, namely leakage, pooling, staining, and blockage that reflect the activity of the CNV complex. ${ }^{1}$ However, FFA and ICG share the inherent risk of invasiveness, which render them impractical in certain clinical situations such as pregnancy, chronic renal failure, and pediatric age group, in addition to the remote but potentially life-threatening complication of anaphylaxis. ${ }^{2,3}$ Hence, the standard of practice in most clinical settings is to limit their use to a minimum possible number of visits despite the indispensable information they provide.
Correspondence: Magdy Moussa Ophthalmology Department, Faculty of Medicine, Medical Campus - Tanta University, Al-Gheish Street, Tanta 3I I I I, Gharbia Governorate, Egypt Tel +20 I2 23I6 I495 Email magdymoussa60@gmail.com 
On the other hand, SD-OCT uses backscattering light intensity to identify different ocular tissues in normal individuals and in various pathological conditions. In patients with CNV, SD-OCT enables noninvasive visualization of the pathological changes in retinal and/or choroidal ultrastructure in response to $\mathrm{CNV}$ formation. In addition, $\mathrm{CNV}$ features such as size, retinal thickness, and associated fluid accumulation could be quantified in numerical values for comparison and assessment of response to therapy. ${ }^{4}$ Nevertheless, SD-OCT cannot differentiate vascular and fibrous components of CNV due to their similar reflectivity properties. In addition, various pathological conditions such as multifocal choroiditis (MFC), central serous chorioretinopathy, and vitelliform dystrophies can cause alteration of the retinal pigment epithelium (RPE) and deposition of sub-retinal heterogenous material with very similar backscattering light intensity, which render them virtually indistinguishable from $\mathrm{CNV}$. Therefore, SD-OCT represents a useful supplement to FFA and ICG in the diagnosis of CNV but cannot replace either of them. ${ }^{5-8}$

The swept-source optical coherence tomography (SSOCT) technology incorporated new features including long wavelength $(1,050 \mathrm{~nm})$ scanning light, less susceptibility to sensitivity roll-off, and ultrahigh-speed image acquisition. These features enabled deeper penetration, superior axial resolution, and generation of ultrahigh-definition B-scan images of the retinal microstructure. ${ }^{9-13}$ Furthermore, the SS-OCT technology incorporated a blood flow detection algorithm, optical coherence tomography angiography ratio analysis (OCTARA), that is capable of visualizing the superficial retinal capillaries and foveal avascular zone, inner and outer retinal vascular plexuses, choriocapillaris, and larger choroidal vasculature in vivo without the need for contrast injection. ${ }^{14}$ Hence, this emerging technology offered noninvasive alternative that has the potential for deferring the disadvantages of FFA and ICG angiography in imaging CNV while providing analogous information regarding the disease activity and spatial relation of the neovascular tissue to the surrounding structures. This notion has been corroborated by reports from several studies on using swept-source optical coherence tomography angiography (SS-OCTA) in different fundus pathologies as an alternative imaging tool to conventional angiography while yielding comparable and reliable information. ${ }^{15-19}$

The aim of this study was to assess the efficacy of SSOCTA in delineating the morphologic features of CNV during activity and quiescence.

\section{Patients and methods}

\section{Patients}

This was a retrospective observational case series in which we reviewed the clinical data and FFA, SS-OCT, and
SS-OCTA images of all consecutive patients diagnosed with CNV in a private practice from September 2015 to October 2016, and compared the findings. The study included patients with CNV secondary to different fundus pathologies. Diagnosis of CNV was based on biomicroscopic examination and confirmed by typical FFA and OCT criteria of CNV. ${ }^{20}$ Patients were excluded from the study whenever they had media opacity that was dense enough to preclude sufficient image quality for reliable interpretation.

\section{Methods}

\section{FFA}

FFA images were obtained using Topcon TRC 50DX fundus camera (Topcon Corporation, Tokyo, Japan).

\section{SS-OCT}

SS-OCT images were acquired using the deep range imaging (DRI) OCT Triton machine (Topcon Corporation). The machine incorporates the swept-source technology, which utilizes infrared $(1,050 \mathrm{~nm})$ laser source and analog-to-digital acquisition mode that minimizes variation in sensitivity with depth (sensitivity roll-off) allowing deeper penetration and superior axial resolution. The infrared laser operates at a scanning speed of 100,000 A-scans/second. This ultrahigh acquisition speed enables dense raster scanning to acquire high volumetric data generating ultrahigh-definition B-scan images.

\section{SS-OCTA and OCTARA algorithm}

OCTARA (Topcon Corporation) is a blood flow detection algorithm that uses decorrelation motion contrast between rapidly repeated SS-OCT B-scans to visualize blood flow in vivo without the need for contrast injection. This OCTA implement benefits from being merged with SS-OCT technology as the deeper penetration of the infrared wavelength allows segmentation of different layers of the ocular fundus. Therefore, it is possible to generate depth-resolved images of the neovascular network which provide accurate information on the vascular profile of the neovascular plexus at baseline and its location in relation to RPE-Bruch's-choriocapillaris complex. Acquired scans are displayed simultaneously as separate "en face" images of retinal microvasculature and choriocapillaris. It is worthy of note that the OCTARA algorithm generates OCTA images by registering B-scan repetition at each scan location then computing a ratiobased result between corresponding image pixels. This method preserves the integrity of the OCT spectrum and does not result in compromised axial resolution, an inherent disadvantage of other OCTA technologies. 


\section{Image acquisition}

OCTA acquisition protocol in the macular region consisted of a $3 \times 3 \mathrm{~mm}$ area centered onto the fovea for maximum resolution of the vascular network composition. Whenever the lesion extended beyond the image border, a $4.5 \times 4.5 \mathrm{~mm}$ area, or a $6 \times 6 \mathrm{~mm}$ area, was used to include the entire extent of the lesion. By default, the integrated software (IMAGEnet 6) deployed automated segmentation to delineate the neovascular lesion and its location in relation to RPEBruch's membrane-choriocapillaris complex. In cases of high axial myopia, significant disorganization of retinal layers as in large pigment epithelial detachment (PED), or sizeable edema or hemorrhage, the integrated automated segmentation feature failed to detect the correct boundaries of the lesion and we had to resort to manual adjustment of the segmentation slab. To perform manual segmentation, the operator manually placed two segmentation lines at sequential depths guided by corresponding SS-OCT B-scan images to reveal the maximum extent of the lesion and its actual location.

All three imaging modalities were performed on the same day. Patients with CNV were categorized into two groups according to FFA and SS-OCT findings regarding the activity of the $\mathrm{CNV}$, as described in the "Group I: active lesion" section. This was characterized by leakage on FFA with or without deep retinal hemorrhage and characteristic fusiform or nodular hyper-reflective lesion related to the RPE on SS-OCT with associated sub- and/or intra-retinal or sub-RPE fluid, as described in the "Group II: inactive lesion" section. This was characterized by the absence of leakage or minimal late leakage on FFA, a hyper-reflective lesion on SS-OCT causing disorganization of retinal layers, and absence of sub- and/or intra-retinal or sub-RPE fluid. SS-OCTA images were then acquired for each group to define the morphological characteristics of each lesion category in terms of shape, and vascular network configuration. Patient selection for enrollment and image interpretation were undertaken by an experienced retina specialist (MM). The current study was conducted in a private ophthalmic center - MEDIC eye center, Tanta, Egypt. To date, our center is not officially affiliated to an institutional review board. Nevertheless, on conducting the study, we adhered strictly to the tenets of Declaration of Helsinki (2013 revision). Moreover, all patients enrolled in the study received thorough explanation of the nature of the imaging modalities entailed in the study and the expected complications of fluorescein angiography. All patients signed an informed consent prior to enrollment. The consent signed by patients included a statement that authorized the authors to publish patients' photos and data compiled during the study for research purposes in an anonymous manner that does not allow identification of the patient.

\section{Results \\ Study population}

The study included 136 eyes of 105 patients (37 men and 68 women), with a mean age of 56 years (range 9-80 years; SD 16.5). CNV was bilateral in 31 patients (29.5\%). Mean best-corrected visual acuity was $0.9 \log$ MAR (range $0-1.5$ $\log$ MAR; SD 0.4). The main primary pathology detected was exudative age-related macular degeneration (AMD) affecting 54 patients $(51.4 \%)$. The $\mathrm{CNV}$ was classified as type I in 20 eyes (15\%), type II in 86 eyes (63\%), and mixed type in 30 eyes $(22 \%)$ (Table 1$)$.

\section{Image analysis}

SS-OCTA images were obtained for 107 (79\%) out of 136 eyes. The remaining cohort of 29 eyes $(21.3 \%)$ was excluded from image analysis. SS-OCTA image acquisition was not possible in this particular cohort due to unsteady

Table I Baseline patient characteristics

\begin{tabular}{ll}
\hline Baseline characteristics & $\mathbf{n}(\%)$ \\
\hline Male & $37(35)$ \\
Female & $68(65)$ \\
Age, years & \\
$<40$ & $20(19)$ \\
$40-50$ & $14(13.3)$ \\
5 I-60 & $23(22)$ \\
$>60$ & $48(46)$ \\
Baseline BCVA, logMAR & \\
0-0.I & $3(2.2)$ \\
$>0.1-0.3$ & $9(7)$ \\
$>0.3-I$ & $83(61)$ \\
$>$ I & $4 I(30)$ \\
CNV laterality & \\
Unilateral & $74(70.4)$ \\
Bilateral & $3 I(29.5)$ \\
CNV type & \\
Type I & $20(15)$ \\
Type II & $86(63)$ \\
Mixed & $30(22)$ \\
Primary pathology & \\
Exudative AMD & $54(51.4)$ \\
High myopia & $42(40)$ \\
Vitelliform macular dystrophy & $3(3)$ \\
MFC & $2(2)$ \\
Birdshot chorioretinopathy & $\mathrm{I}(1)$ \\
Juvenile foveoschisis & $\mathrm{I}(1)$ \\
Choroidal osteoma & $\mathrm{I}(1)$ \\
Juxta-foveal telangiectasia & $\mathrm{I}(\mathrm{I})$ \\
\hline
\end{tabular}

Abbreviations: AMD, age-related macular degeneration; BCVA, best-corrected visual acuity; CNV, choroidal neovascular membrane; logMAR, logarithm of the minimum angle of resolution; MFC, multifocal choroiditis; N, number. 
fixation caused by lack of patient cooperation or by poor vision due to advanced macular pathology. Motion artifacts were detected in 32 eyes (30\%). These artifacts degraded the image quality, although they remained readable with unequivocal display of hyperintense signal of blood flow within CNV lesions.

\section{Group I: active lesion}

Features detected on SS-OCTA images of active CNV lesion were increased density of tiny capillaries, extensive arborization, vascular anastomosis and looping within the $\mathrm{CNV}$, and a hollow zone around the lesion separating it from the surrounding normal vasculature (Figures 1 and 2). All CNV lesions in this group showed signs of activity on FFA and on SS-OCT.

\section{Group II: inactive lesion}

Features detected on SS-OCTA images of inactive CNV lesion were decreased capillary density, presence of large linear vessels widely separated by dark spaces with no or minimal anastomosis, and presence of feeder vessels either single, double, or multiple emanating from a main trunk and supplying the neovascular complex (Figures 3 and 4). All $\mathrm{CNV}$ lesions in this group lacked signs of activity on FFA and on SS-OCT.

\section{Anatomical location of the CNV}

All type I CNV lesions were best visualized on the SS-OCTA en face projection of the choriocapillaris (Figure 5), whereas all type II CNV lesions were best visualized on the SS-OCTA en face projection of the outer retina (Figure 6). Mixed CNV lesions were visualized on both modes with maximum visualization on either outer retina or choriocapillaris projection depending on whether the main bulk of the lesion was located above or beneath the RPE.

\section{Correlation between FFA, OCT, and OCTA}

SS-OCTA images demonstrated high sensitivity in detecting CNV lesion. We detected positive correlation between SS-OCTA, FFA, and SS-OCT images in 104 eyes (97\%)
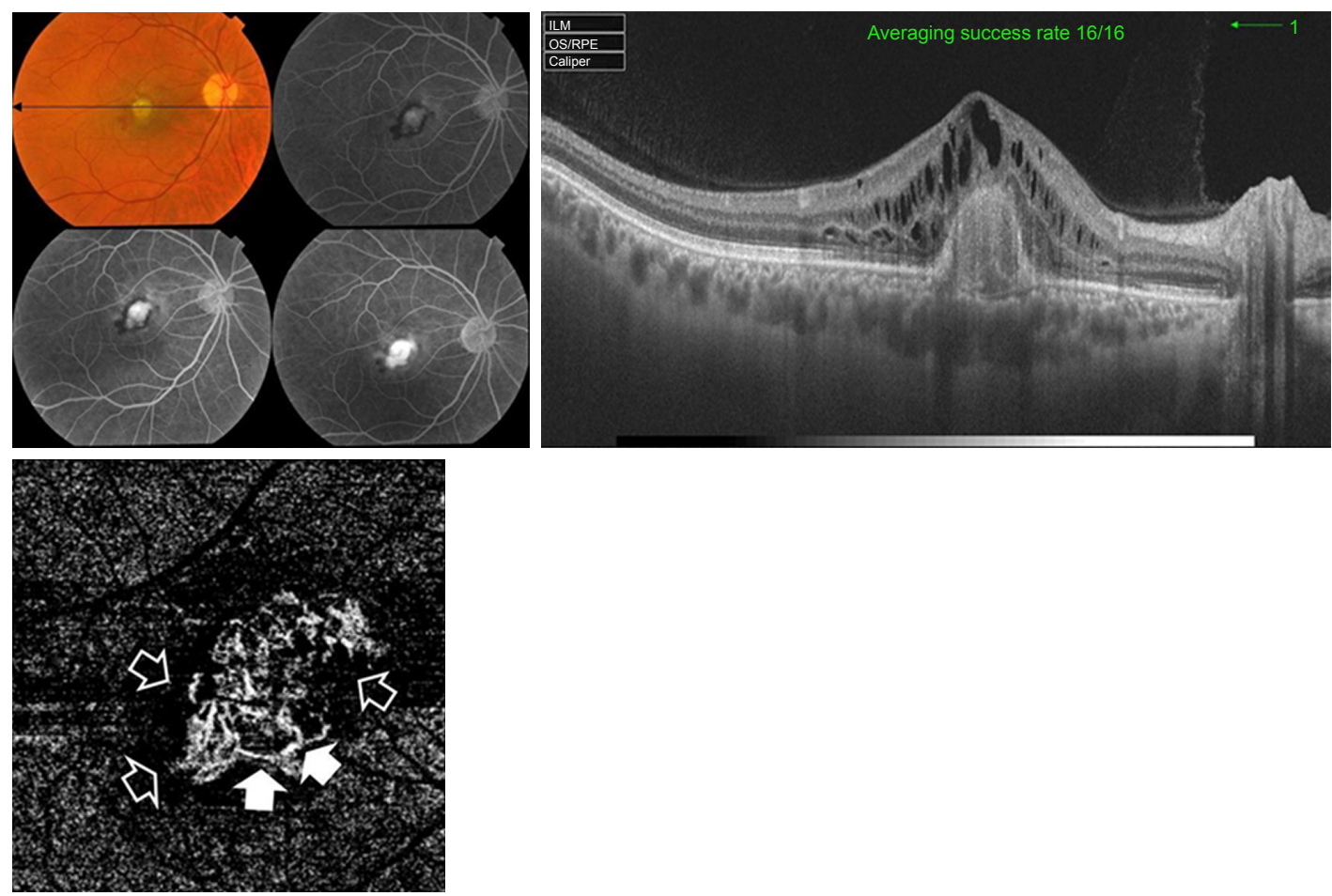

Figure I Upper left, color photo and FFA of the right eye of a 55-year-old male with exudative AMD and classic CNV. UPper right, corresponding SS-OCT image in radial scan mode shows hyper-reflective nodular lesion located entirely above the RPE. The lesion resulted in disruption of the retinal layers and is associated with gross intra-retinal edema which indicates type II active CNV. Lower left, "en face" SS-OCTA image of the same eye taken at the level of the outer retina in a $3 \times 3 \mathrm{~mm}$ field. The neovascular complex is displayed as a hyperintense signal caused by increased blood flow within the lesion. The remaining avascular outer retina generates a hypointense signal due to absent blood flow and is displayed as a dark-gray background. The lesion demonstrates homogenous network of tiny interlacing capillaries with occasional larger vessels displayed as jet-white streaks (closed arrow heads). The entire complex is surrounded by a dark lucid interval intervening between the lesion and the surrounding normal outer retina (open arrow heads).

Abbreviations: AMD, age-related macular degeneration; CNV, choroidal neovascular membrane; FFA, fundus fluorescein angiography; OCT, optical coherence tomography; OCTA, OCT angiography; RPE, retinal pigment epithelium; SS-OCT, swept-source OCT; SS-OCTA, swept-source OCTA. 

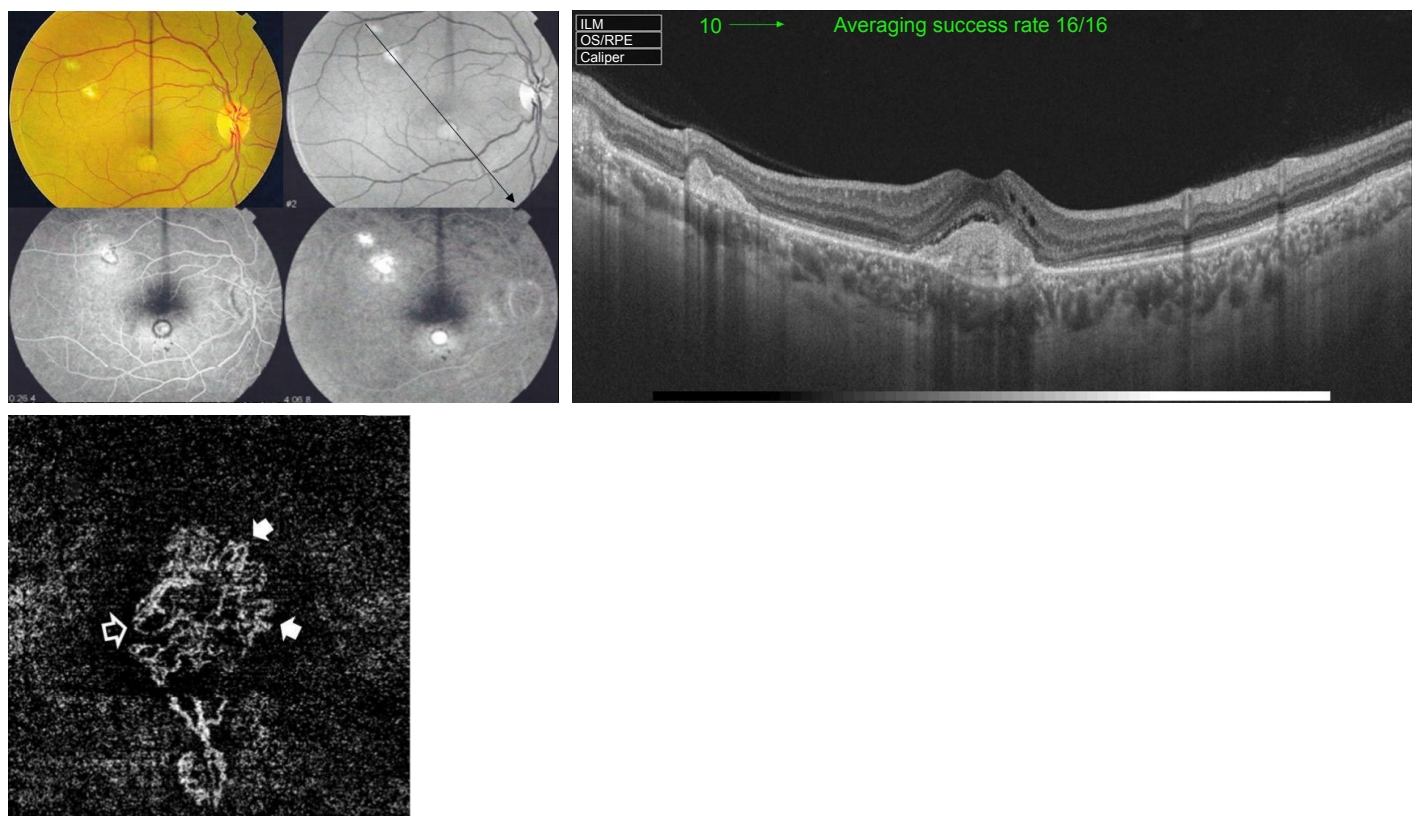

Figure 2 Upper left, color photo, red-free photo, and FFA of the right eye of a 5 I-year-old female with classic CNV on top of MFC. UPper right, corresponding SS-OCT image in line scan mode shows three hyper-reflective nodular lesions located entirely above the RPE. The sub-foveal lesion resulted in disruption of the outer retinal layers and is associated with overlying intra-retinal edema which indicates type II active CNV. Lower left, "en face" SS-OCTA image of the same eye taken at the level of the outer retina in a $3 \times 3 \mathrm{~mm}$ field. The central portion of the lesion demonstrates dense arborization. More peripherally, several vascular anastomosis (closed arrow heads) and looping (open arrow head) are shown.

Abbreviations: CNV, choroidal neovascular membrane; FFA, fundus fluorescein angiography; MFC, multifocal choroiditis; OCT, optical coherence tomography; OCTA, OCT angiography; RPE, retinal pigment epithelium; SS-OCT, swept-source OCT; SS-OCTA, swept-source OCTA.
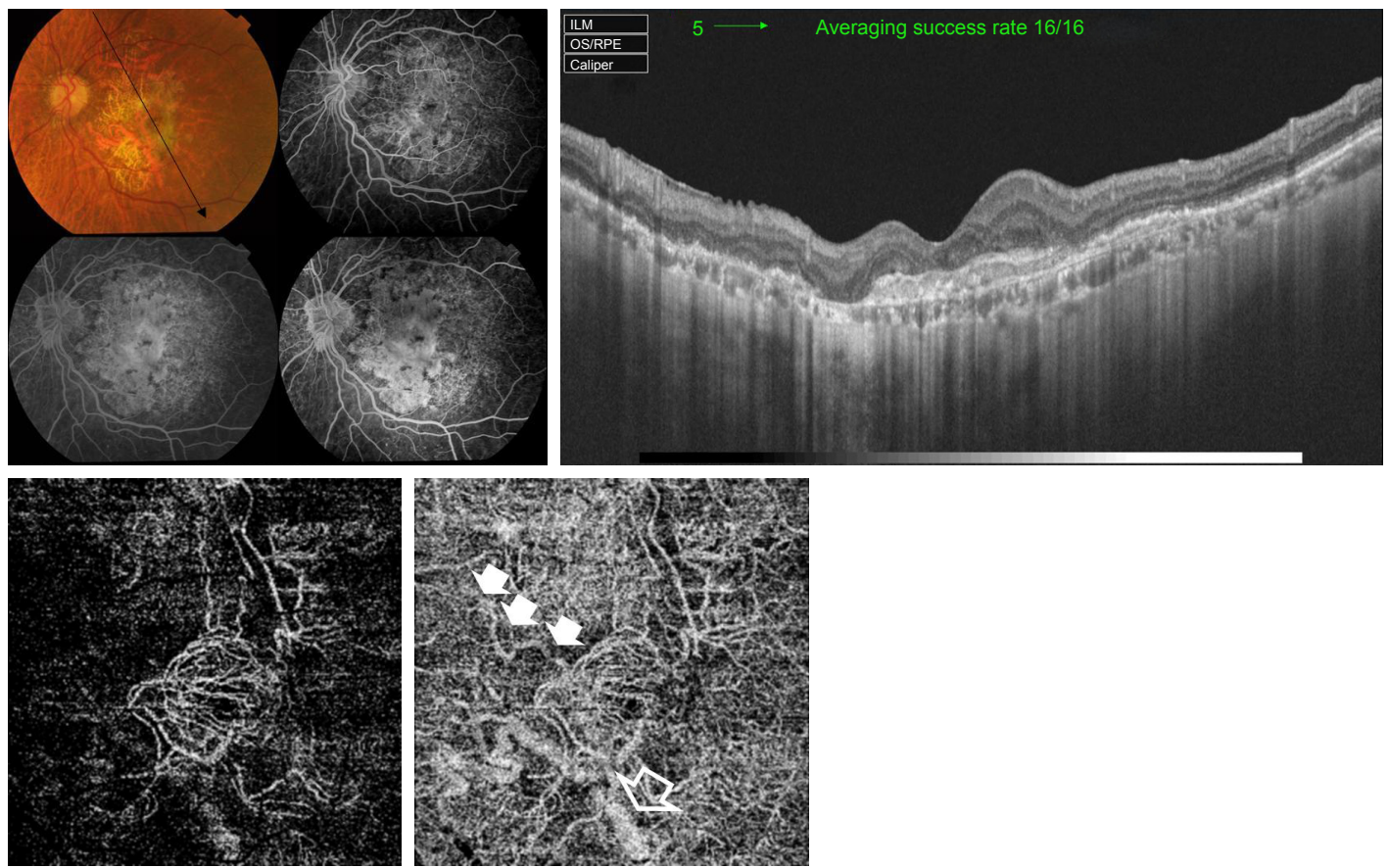

Figure 3 Upper left, color photo and FFA of the left eye of a 75-year-old female with scarred CNV secondary to AMD. UPper right, corresponding SS-OCT image in radial scan mode shows marked disorganization of the outer retina with marked thinning of the RPE and choriocapillaris. No intra-retinal fluid is discernible. Lower, "en face" SSOCTA image of the same eye taken at the level of the outer retina (left) and the choriocapillaris (right) in a $6 \times 6 \mathrm{~mm}$ field. The entire lesion complex is composed mostly of large mature vessels that yield hyperintense signal with intervening hollowness due to absent capillaries. A single large feeder vessel is shown supplying the main lesion (closed arrow heads). The shadow of the large choroidal vessel (open arrow head) is shown.

Abbreviations: AMD, age-related macular degeneration; CNV, choroidal neovascular membrane; FFA, fundus fluorescein angiography; OCT, optical coherence tomography; OCTA, OCT angiography; RPE, retinal pigment epithelium; SS-OCT, swept-source OCT; SS-OCTA, swept-source OCTA. 

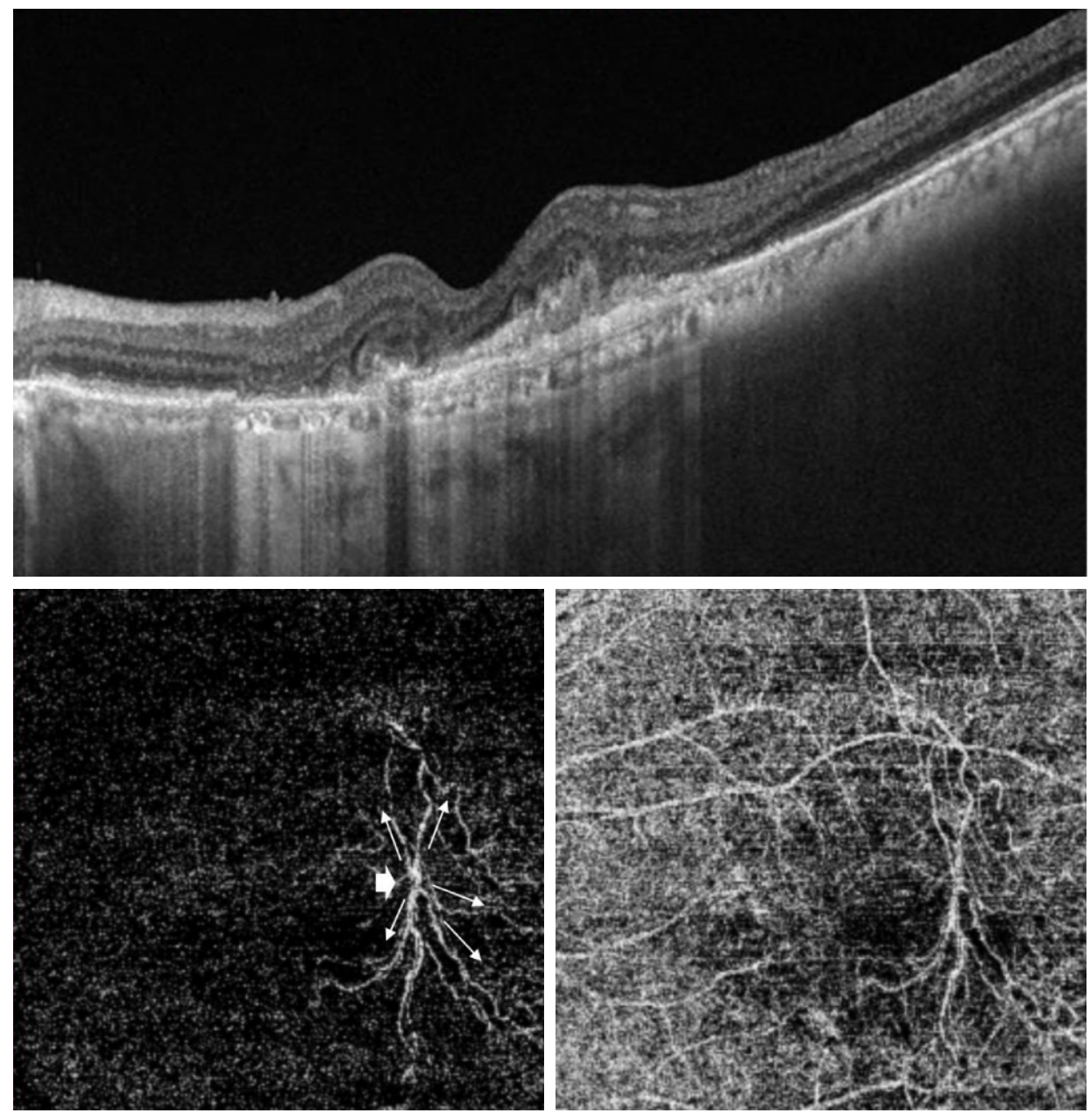

Figure 4 Upper, SS-OCT of the left eye of an 80-year-old male with AMD. Marked disorganization of the outer retina with marked thinning of the RPE and choriocapillaris and minimal intra-retinal fluid accumulation are shown. Lower, corresponding "en face" SS-OCTA image taken at the level of the outer retina (left) and the choriocapillaris (right) in a $6 \times 6 \mathrm{~mm}$ field. The medusa head configuration composed of at least five large mature vessels (arrows) emanating from single main trunk (arrow head) is shown. Abbreviations: AMD, age-related macular degeneration; OCT, optical coherence tomography; OCTA, OCT angiography; RPE, retinal pigment epithelium; SS-OCT, sweptsource OCT; SS-OCTA, swept-source OCTA.

out of 107 eyes. In the remaining three eyes (3\%), SS-OCTA confirmed the absence of CNV, whereas FFA and SS-OCT either were inconclusive in the diagnosis of CNV or yielded false-positive results (Figures 7 and 8).

\section{Discussion}

The current consensus for the management of CNV entails dual imaging using FFA and/or ICG combined with OCT to obtain functional and anatomical information on the lesion that help to establish the diagnosis, to predict the visual outcome of therapy, and to tailor a follow-up protocol. FFA reflects the activity of CNV by depicting different patterns of dye leakage, whereas ICG is crucial in identifying the entire extent of the lesion especially in type I CNV. The superior axial resolution of OCT provided anatomical information on the location of the $\mathrm{CNV}$ within the retina and choroid in relation to neighboring structures and changes in retinal microstructure secondary to the presence of the CNV, accumulation of fluid, and/or hemorrhage. ${ }^{1}$

OCTA recently emerged as a noninvasive imaging modality that has the potential for replacing FFA/ICG to complement OCT in CNV imaging, thereby defraying the need for using intravenous dye injection. By using decorrelation motion contrast, this technology is able to detect intra-lesional blood flow in vivo and generate flow patterns that reflect the activity of the neovascular complex, thereby representing a noninvasive substitute that provides the functional information obtained from dye-based angiography. ${ }^{15-17}$

The current series was able to confirm this notion by demonstrating specific blood flow patterns within the CNV in both active and quiescent forms. Our results concur with several authors who used OCTA technology to describe blood flow 

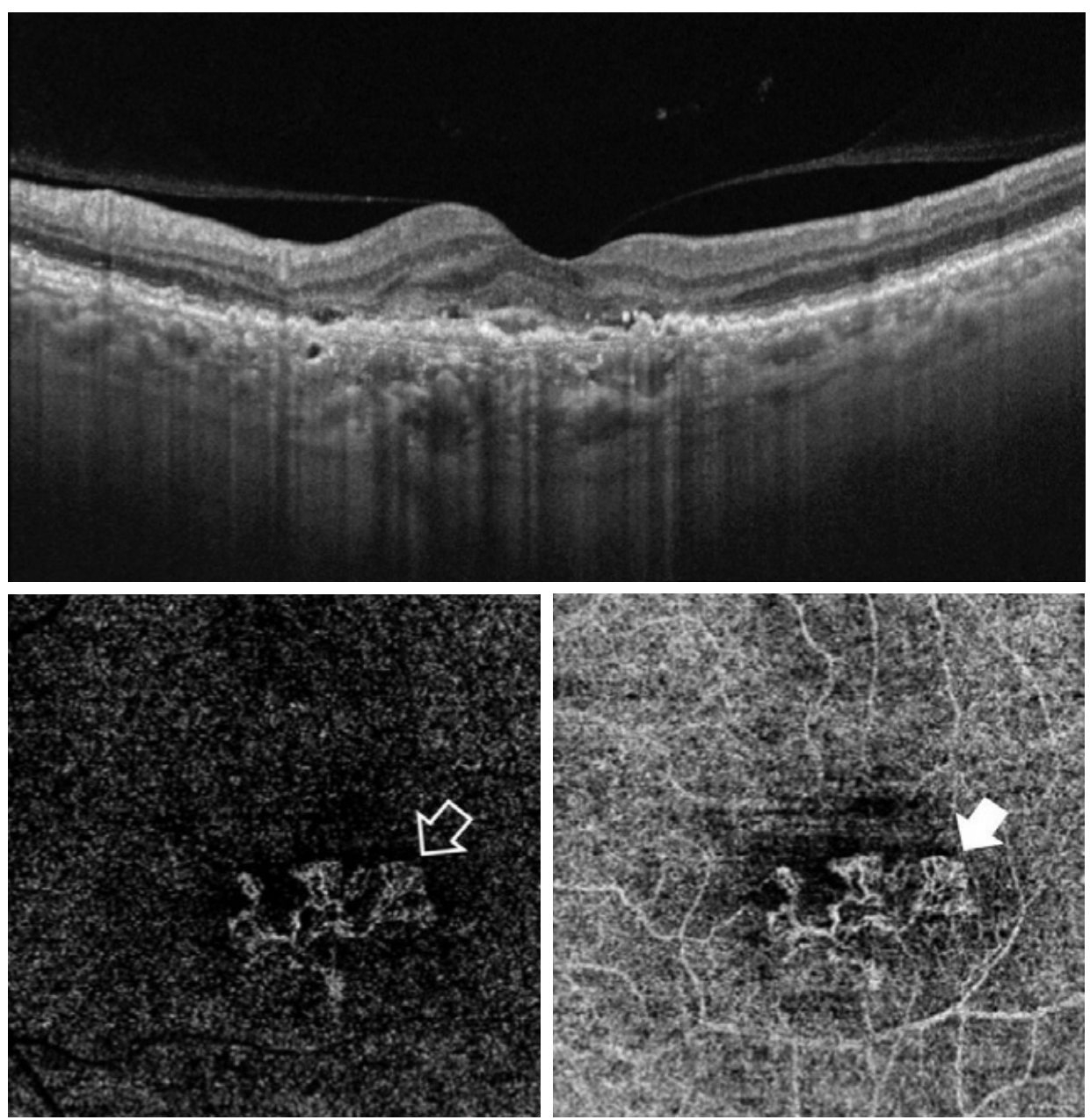

Figure 5 Upper, SS-OCT image of the left eye of a 66-year-old female with neovascular AMD shows multiple RPE detachments. The RPE in the sub-foveal area is irregular, thickened, and raised-up by a moderately hyper-reflective lesion associated with disorganization of the outer retina and accumulation of sub- and intra-retinal fluid, which indicates type I CNV. Lower, "en face" SS-OCTA image captured at the level of the outer retina (left) and choriocapillaris (right) in a $3 \times 3$ mm field. The extent of arborization of the neovascular complex is most revealed at the choriocapillaris projection (closed arrow head) compared to the outer retina projection (open arrow head).

Abbreviations: AMD, age-related macular degeneration; CNV, choroidal neovascular membrane; OCT, optical coherence tomography; OCTA, OCT angiography; RPE, retinal pigment epithelium; SS-OCT, swept-source OCT; SS-OCTA, swept-source OCTA.

patterns within CNV. Coscas et $\mathrm{al}^{21}$ conducted a prospective study of 80 eyes with $\mathrm{CNV}$ and classified the lesion according to the morphologic characteristics of the vascular network into patterns of activity and quiescence based on OCTA images. For active lesions, these authors coined specific terminology as lacy wheel or sea fan to describe arborization within CNV and dead tree to describe large mature vessels indicative of inactive lesion. Similarly, El Ameen et $\mathrm{al}^{22}$ described in a prospective series of 14 eyes with type II CNV, specific morphological patterns using OCTA images. The authors described the active vascular network as medusashaped lesion or glomerulus-shaped lesion. Conversely, Kuehlewein et $\mathrm{al}^{23}$ in a prospective series of 33 eyes with inactive type I CNV in AMD used same terms, sea fan and medusa patterns, to describe different morphological patterns of large caliber mature feeder vessels and their branches as indicative of inactive lesions. Miere et $\mathrm{al}^{24}$ and Dansingani et $\mathrm{al}^{25}$ reported the morphological characteristics of type III CNV using OCTA in 18 eyes and in two eyes, respectively.

In our study, we detected a dark area surrounding active CNV lesions and that was displayed as a hypointense clear zone on SS-OCTA images. This finding is consistent with several authors ${ }^{21,23,26,27}$ who reported the same sign. A plausible explanation for this sign is that CNV tends to develop amid a region of choriocapillaris alteration caused by impaired flow to compensate for ischemia. The region of choriocapillaris alteration is located underneath the CNV and extends beyond its margins in the form of a ring or halo and appears as hypointense or silent area on SS-OCTA images due to reduced blood flow. 

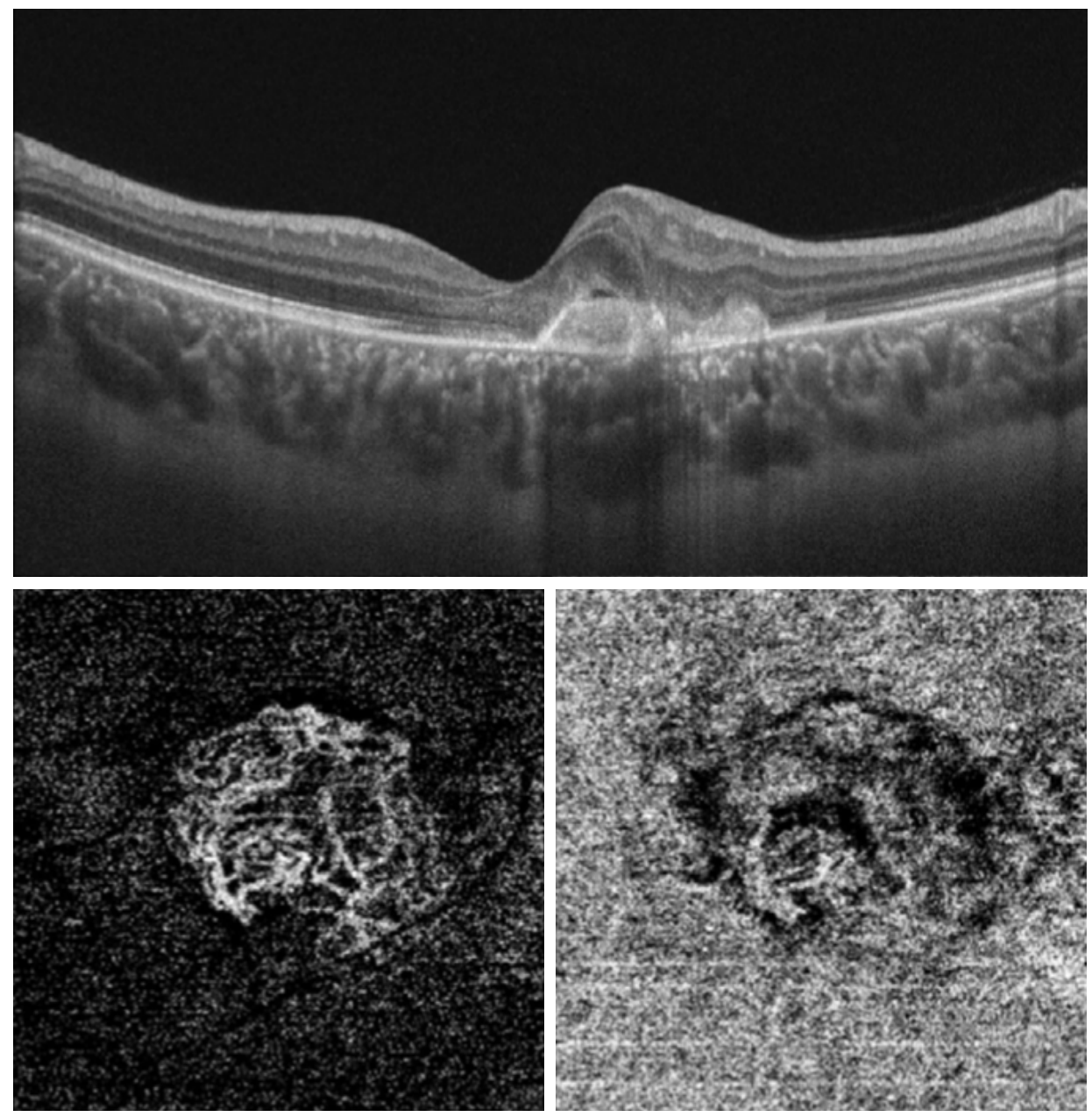

Figure 6 Upper, SS-OCT of the left eye of a 30-year-old female with MFC shows hyper-reflective double hump lesion causing disorganization of the outer retina and is associated with intra-retinal fluid. The lesion is located entirely above the RPE, which indicates type II CNV. Lower, "en face" SS-OCTA image captured at the level of the outer retina (left) and choriocapillaris (right) in a $3 \times 3 \mathrm{~mm}$ field. The outer boundaries of the lesion and the arborizing pattern of the neovascular network are best delineated in the outer retina projection, whereas in the choriocapillaris projection the boundaries of the lesion are indistinguishable from the surrounding background in several locations.

Abbreviations: CNV, choroidal neovascular membrane; MFC, multifocal choroiditis; OCT, optical coherence tomography; OCTA, OCT angiography; RPE, retinal pigment epithelium; SS-OCT, swept-source OCT; SS-OCTA, swept-source OCTA.

In terms of efficacy of SS-OCTA compared to FFA and SS-OCT, the current study found that SS-OCTA had high sensitivity index in detecting CNV lesions. In our series, SS-OCTA corresponded to FFA and SS-OCT in $97 \%$ of cases. Coscas et $\mathrm{al}^{21}$ found that OCTA corresponded to FFA, ICG, and SD-OCT in $>90 \%$ of their patients. Jia et $\mathrm{al}^{26}$ used SS-OCTA prototype to detect CNV in five patients with neovascular AMD confirmed by FFA. These authors reported that OCTA successfully detected CNV in all cases (100\%). Moult et a ${ }^{27}$ reported successful detection of CNV by OCTA in 16 out of 17 eyes (94\%) with neovascular AMD confirmed by FFA and ICG. However, De Carlo et $\mathrm{al}^{28}$ reported 50\% sensitivity of OCTA in detecting CNV. These authors stated that the remaining $50 \%$ of their patients in whom OCTA yielded false-negative results had large amounts of motion artifact in one patient and dense sub-retinal hemorrhage that blocked the SD-OCT signal in three patients. The authors proposed that the increased penetrance of SS-OCT or enhanced depth imaging (EDIOCT) might improve visualization of $\mathrm{CNV}$ in cases obscured by dense hemorrhage. However, Moult et al ${ }^{27}$ reported in their series that the single case in which OCTA revealed false-negative result had dense subretinal hemorrhage that caused severe attenuation of the SS-OCT signal.

In the current study, we found that whenever there was concordance between the three imaging modalities tested in CNV diagnosis, SS-OCTA excelled in providing detailed depiction of the neovascular network compared to FFA and SS-OCT. However, whenever FFA and SS-OCT were inconclusive or revealed false-positive results, SS-OCTA was decisive in excluding CNV. Two of our patients had sub-foveal hemorrhage that caused blocked 

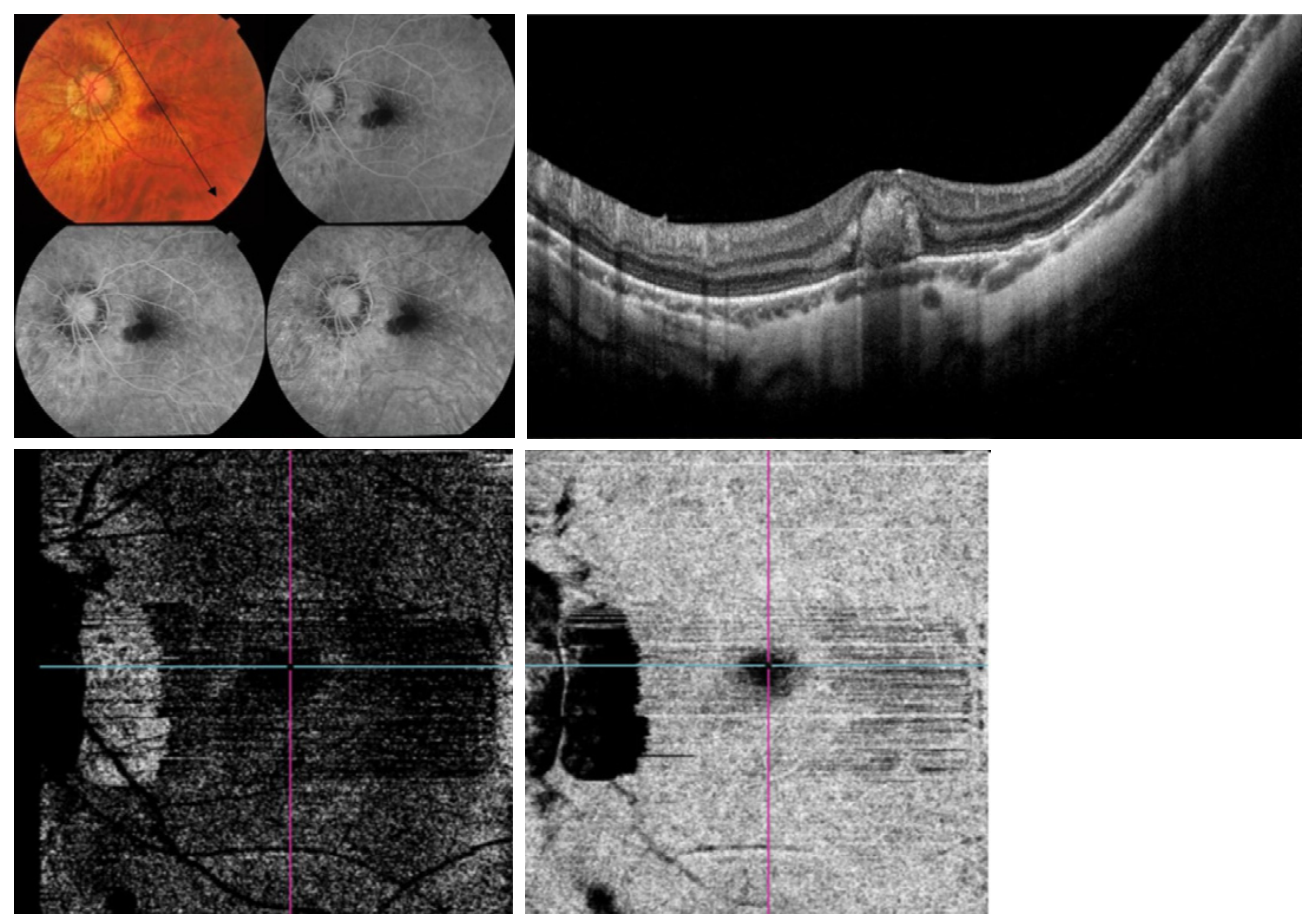

Figure 7 Upper left, color photo and FFA of the left eye of a 32-year-old female with high myopia and sub-foveal hemorrhage. The blocked fluorescence on FFA in the foveal region is shown. Upper right, corresponding SS-OCT image in a radial scan mode shows hyper-reflective sub-foveal lesion corresponding to the hemorrhage seen in color photo and FFA. Both, FFA and SS-OCT are inconclusive for CNV development. Lower, "en face" SS-OCTA image captured at the level of the outer retina in a $6 \times 6$ mm field (left) and co-registered OCT segmentation slabs (right). There is no identifiable decorrelation signal characteristic of abnormal vascular network formation in the area of suspicious CNV formation.

Abbreviations: CNV, choroidal neovascular membrane; FFA, fundus fluorescein angiography; OCT, optical coherence tomography; OCTA, OCT angiography; SS-OCT, swept-source OCT; SS-OCTA, swept-source OCTA.
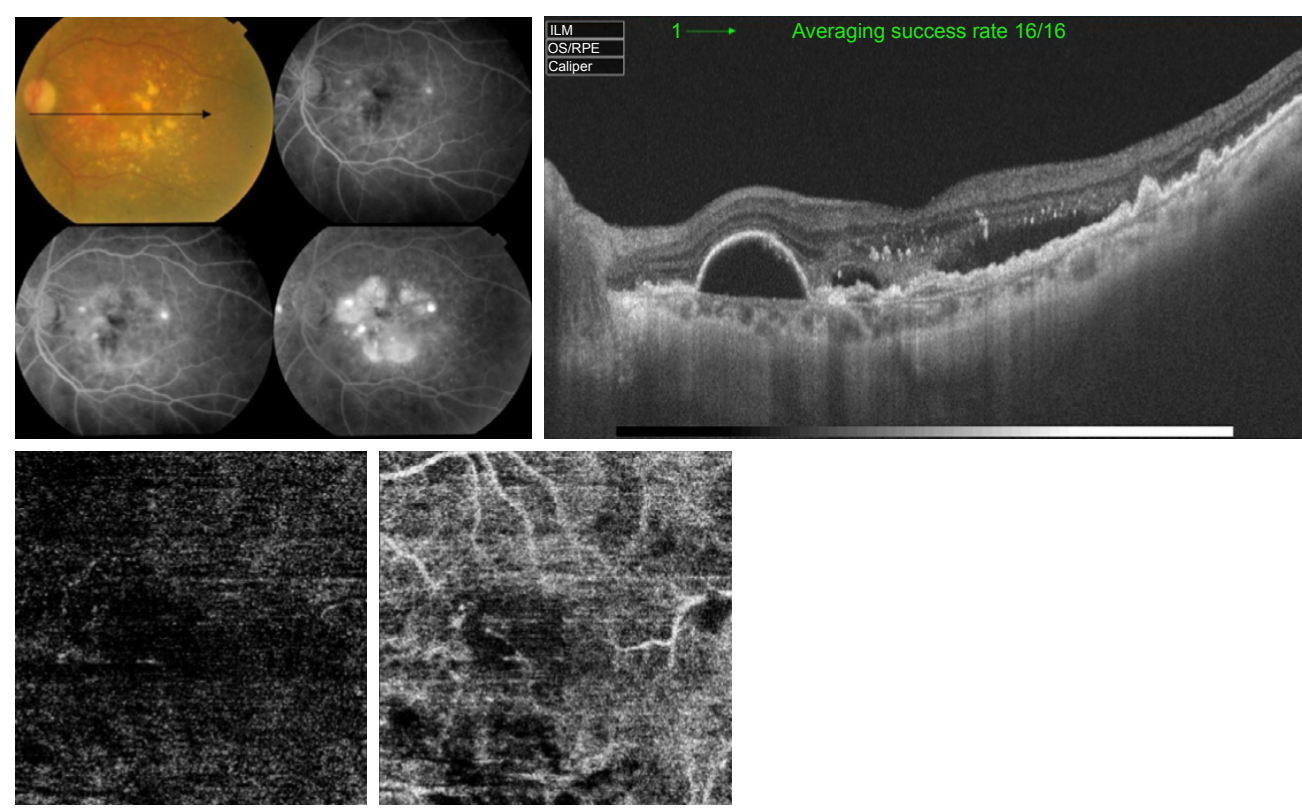

Figure 8 Upper left, color photo and FFA of the left eye of a 66-year-old female with intermediate AMD and extensive drusen. The late frames of FFA show pooling in multiple PEDs and LLIO characteristic of occult CNV. UPper right, corresponding SS-OCT image in radial scan mode shows RPE detachment. The RPE is irregular, thickened, and raised-up by a moderately hyper-reflective lesion with accumulation of sub-retinal fluid, suggestive of type I CNV formation. Lower, "en face" SS-OCTA image of the same eye taken at the level of the outer retina in a $3 \times 3 \mathrm{~mm}$ field (left) and co-registered OCT segmentation slabs (right). The absence of identifiable decorrelation signal characteristic of abnormal vascular network formation in the area of suspicious CNV formation is shown.

Abbreviations: AMD, age-related macular degeneration; CNV, choroidal neovascular membrane; FFA, fundus fluorescein angiography; LLIO, late leakage of indeterminate origin; OCT, optical coherence tomography; OCTA, OCT angiography; PED, pigment epithelial detachment; RPE, retinal pigment epithelium; SS-OCT, swept-source OCT; SS-OCTA, swept-source OCTA. 
fluorescence on FFA and signal attenuation on SS-OCT. In these two patients, SS-OCTA confirmed the absence of CNV (Figure 7). A third patient had intermediate AMD and extensive drusen formation and in whom FFA and SS-OCT were highly suspicious of occult CNV. SS-OCTA confirmed the absence of decorrelation signal characteristic of active CNV (Figure 8). Likewise, Kotsolis et $\mathrm{al}^{29}$ reported in a retrospective analysis of 20 eyes with $\mathrm{CNV}$ secondary to MFC that they were unable to determine the type of CNV using conventional FFA due to overlying sub-retinal hemorrhage. Levison et $\mathrm{al}^{5}$ conducted a prospective case series of 12 patients with CNV secondary to punctate inner choroidopathy and MFC. These authors found that OCTA identified $\mathrm{CNV}$ in all cases in which FFA was inconclusive.

The current study had a number of limitations that should be considered while interpreting the results reported therein. Limitations inherent to the study included its retrospective design that caused inter- and intra-group heterogeneity in important aspects including stratification of different pathologies, CNV types, and CNV stages. In addition, the study allowed recruitment of patients with CNV regardless whether the CNV was naive or pretreated and even though with variable treatment course and regimen in pretreated patients. Additional limitations inherent to SS-OCTA technology included motion artifacts that degraded the image quality in almost $30 \%$ of our patients. Motion artifacts had been reported in a number of studies as a significant disadvantage during image acquisition. ${ }^{19,23,28,30}$ Technology development focused on improved eye tracking and faster acquisition time could overcome this limitation. Another important limitation was the automated segmentation errors in cases of high myopia, presence of large amounts of edema and/or hemorrhage, and large PEDs. In those cases, we had to resort to manual segmentation performed by the operator which could be a source of bias. Future development of segmentation algorithms is needed to overcome this obstacle.

\section{Conclusion}

Swept-source technology including SS-OCT and SS-OCTA is a reproducible risk-free analog for conventional FFA in imaging CNV. Combined SS-OCT and SS-OCTA are capable of providing accurate anatomical information on the location of the CNV and associated secondary changes in the retinal architecture and functional information in the form of characteristic morphological patterns that reflect the disease activity based on blood flow within the CNV. SS-OCTA is particularly versatile in cases, where FFA and SS-OCT are inconclusive.

\section{Disclosure}

None of the authors have proprietary interest in any material used in this study. The authors report no other conflicts of interest in this work.

\section{References}

1. Bressler NM, Bressler SB. Neovascular (exudative or 'wet') age-related macular degeneration. In: Ryan SJ, editor. Retina. 5 ed. Philadelphia: Saunders; 2013:1183-1210.

2. Richard G, Soubrane G, Yannuzzi LA. The principles of fluorescein angiography. In: Richard G, editor. Fluorescein and ICG Angiography. Textbook and Atlas. 2 ed. New York: Thieme; 1998:1-9.

3. Johnson RN, Fu AD, McDonald HR, et al. Fluorescein angiography basic principles and interpretation. In: Ryan SJ, editor. Retina. 5 ed. Philadelphia: Saunders; 2013:2-50.

4. Liu R, Li J, Li Z, et al. Distinguishing polypoidal choroidal vasculopathy from typical neovascular age-related macular degeneration based on spectral domain optical coherence tomography. Retina. 2016;36(4): 778-786.

5. Levison AL, Baynes KM, Lowder CY, Kaiser PK, Srivastava SK. Choroidal neovascularisation on optical coherence tomography angiography in punctate inner choroidopathy and multifocal choroiditis. $\mathrm{BrJ}$ Ophthalmol. 2017;101(5):616-622.

6. Lupidi M, Coscas G, Cagini C, Coscas F. Optical coherence tomography angiography of a choroidal neovascularization in adult onset foveomacular vitelliform dystrophy: pearls and pitfalls. Invest Ophthalmol Vis Sci. 2015;56(13):7638-7645.

7. Querques G, Zambrowski O, Corvi F, et al. Optical coherence tomography angiography in adult-onset foveomacular vitelliform dystrophy. Br J Ophthalmol. 2016;100(12):1724-1730.

8. Castillo MM, Mowatt G, Lois N, et al. Optical coherence tomography for the diagnosis of neovascular age-related macular degeneration: a systematic review. Eye. 2014;28(12):1399-1406.

9. Bonnin S, Mané V, Couturier A, et al. New insight into the macular deep vascular plexus imaged by optical coherence tomography angiography. Retina. 2015;35(11):2347-2352.

10. Grulkowski I, Liu JJ, Potsaid B, et al. Retinal, anterior segment and full eye imaging using ultrahigh speed swept source OCT with vertical-cavity surface emitting lasers. Biomed Opt Express. 2012;3(11):2733-2751.

11. Gao SS, Liu G, Huang D, Jia Y. Optimization of the split-spectrum amplitude-decorrelation angiography algorithm on a spectral optical coherence tomography system. Opt Lett. 2015;40(10):2305-2308.

12. Kuehlewein L, Tepelus TC, An L, Durbin MK, Srinivas S, Sadda SR. Noninvasive visualization and analysis of the human parafoveal capillary network using swept source OCT optical microangiography. Invest Ophthalmol Vis Sci. 2015;56(6):3984-3988.

13. Savastano MC, Lumbroso B, Rispoli M. In vivo characterization of retinal vascularization morphology using optical coherence tomography angiography. Retina. 2015;35(11):2196-2203.

14. Stanga PE, Tsamis E, Papayannis A, Stringa F, Cole T, Jalil A. Sweptsource optical coherence tomography angiography ${ }^{\mathrm{TM}}$ (Topcon Corp, Japan): technology review. Dev Ophthalmol. 2016;56:13-17.

15. Spaide RF. Volume-rendered angiographic and structural optical coherence tomography. Retina. 2015;35(11):2181-2187.

16. De Carlo TE, Romano A, Waheed NK, Duker JS. A review of optical coherence tomography angiography (OCTA). Int J Ret Vit. 2015;1:5.

17. Novais EA, Adhi M, Moult EM, et al. Choroidal neovascularization analyzed on ultrahigh-speed swept-source optical coherence tomography angiography compared to spectral-domain optical coherence tomography angiography. Am J Ophthalmol. 2016;164:80-88.

18. Inoue M, Balaratnasingam C, Freund KB. Optical coherence tomography angiography of polypoidal choroidal vasculopathy and polypoidal choroidal neovascularization. Retina. 2015;35(11):2265-2274.

19. Kuehlewein L, Dansingani KK, De Carlo TE, et al. Optical coherence tomography angiography of type 3 neovascularization secondary to age-related macular degeneration. Retina. 2015;35(11):2229-2235. 
20. AMD Guidelines Group. Age-Related Macular Degeneration: Guidelines for Management. The Royal College of Ophthalmologists; London, 2013:1-45.

21. Coscas GJ, Lupidi M, Coscas F, Cagini C, Souied EH. Optical coherence tomography angiography versus traditional multimodal imaging in assessing the activity of exudative age-related macular degeneration. Retina. 2015;35:2219-2228.

22. El Ameen A, Cohen SY, Semoun O, et al. Type 2 neovascularization secondary to age-related macular degeneration imaged by optical coherence tomography angiography. Retina. 2015;35(11):2212-2218.

23. Kuehlewein L, Bansal M, Lenis TL, et al. Optical coherence tomography angiography of type 1 neovascularization in age-related macular degeneration. Am J Ophthalmol. 2015;160(4):739-748.

24. Miere A, Querques G, Semoun O, et al. Optical coherence tomography angiography in early type 3 neovascularization. Retina. 2015;35: 2236-2241.

25. Dansingani KK, Naysan J, Freund KB. En face OCT angiography demonstrates flow in early type 3 neovascularization (retinal angiomatous proliferation). Eye. 2015;29(5):703-706.
26. Jia Y, Bailey ST, Wilson DJ, et al. Quantitative optical coherence tomography angiography of choroidal neovascularization in age-related macular degeneration. Ophthalmology. 2014;121(7):1435-1444.

27. Moult E, Choi W, Waheed NK, et al. Ultrahigh-speed swept-source OCT angiography in exudative AMD. Ophthalmic Surg Lasers Imaging Retina. 2014;45(6):496-505.

28. De Carlo TE, Filho MAB, Chin AT, et al. Spectral-domain optical coherence tomography angiography of choroidal neovascularization. Ophthalmology. 2015;122(6):1228-1238.

29. Kotsolis AI, Killian FA, Ladas ID, Yannuzzi LA. Fluorescein angiography and optical coherence tomography concordance for choroidal neovascularization in multifocal choroiditis. $\mathrm{Br}$ J Ophthalmol. 2010; 94:1506-1508.

30. Spaide RF, Fujimoto JG, Waheed NK. Image artifacts in optical coherence tomography angiography. Retina. 2015;35(11):2163-2180.
Clinical Ophthalmology

\section{Publish your work in this journal}

Clinical Ophthalmology is an international, peer-reviewed journal covering all subspecialties within ophthalmology. Key topics include: Optometry; Visual science; Pharmacology and drug therapy in eye diseases; Basic Sciences; Primary and Secondary eye care; Patien Safety and Quality of Care Improvements. This journal is indexed on

Submit your manuscript here: http://www.dovepress.com/clinical-ophthalmology-journal

\section{Dovepress}

PubMed Central and CAS, and is the official journal of The Society of Clinical Ophthalmology (SCO). The manuscript management system is completely online and includes a very quick and fair peer-review system, which is all easy to use. Visit http://www.dovepress.com/ testimonials.php to read real quotes from published authors. 